\title{
Friendship Dynamics: Modelling Social Relationships through a Fuzzy Agent-Based Simulation
}

\author{
Samer Hassan, ${ }^{1,2}$ Mauricio Salgado, ${ }^{2}$ and Juan Pavón ${ }^{1}$ \\ ${ }^{1}$ Grupo de Investigación en Agentes Software: Ingeniería y Aplicaciones (GRASIA), \\ Departamento de Ingeniería del Software e Inteligencia Artificial, Universidad Complutense Madrid, \\ 28040 Madrid, Spain \\ ${ }^{2}$ Centre for Research in Social Simulation (CRESS), Department of Sociology, University of Surrey, \\ Guildford, Surrey GU2 7XH, UK
}

Correspondence should be addressed to Samer Hassan, samer@fdi.ucm.es

Received 11 March 2011; Accepted 9 June 2011

Academic Editor: Guang Zhang

Copyright (C) 2011 Samer Hassan et al. This is an open access article distributed under the Creative Commons Attribution License, which permits unrestricted use, distribution, and reproduction in any medium, provided the original work is properly cited.

Social relationships such as friendship and partner choice are ruled by the proximity principle, which states that the more similar two individuals are, the more likely they will become friends. However, proximity, similarity, and friendship are concepts with blurred edges and imprecise grades of membership. This study shows how to simulate these friendship dynamics in an agent-based model that applies fuzzy sets theory to implement agent attributes, rules, and social relationships, explaining the process in detail. Although in principle it may be thought that the use of fuzzy sets theory makes agent-based modelling more elaborated, in practice it saves the modeller from taking some arbitrary decisions on how to use crisp values for representing properties that are inherently fuzzy. The consequences of applying fuzzy sets and operations to define a fuzzy friendship relationship are compared with a simpler implementation, with crisp values. By integrating agent computational models and fuzzy set theory, this paper provides useful insights into scholars and practitioners to tackle the uncertainty inherent to social relationships in a systematic way.

\section{Introduction}

The dynamics of social relationships, such as friendship or partnership patterns, is a complex field of study. In this context, social dynamics refers to the collection of agent interactions, their conditions, and associated mechanisms, together with the emergent behaviour that they bring about. The field has attracted a great deal of interest over the past decades, mostly due to a very rapid increase in data availability and better modelling techniques. A large body of sociological and psychological research has shown that individuals' actions, even those 
actions that would be prima facie based just on personal preferences, are often influenced by the people they interact with. Several researchers have highlighted the importance of the significant others' actions for explaining a vast array of social relationships, including friendship choice [1], romantic networks [2], virginity pledges [3], sexual behaviour [4], contraceptive use [5], and the risk of divorce [6]. Nowadays, there is a fairly detailed picture of many characteristics of individuals and contextual factors that influence friendship choice and romantic networks, such as social proximity, similarity, and individual characteristics of the available acquaintances, among others. However, despite the advances in the field, two important issues are still open. At the methodological level, there are few dynamic models in the literature that address these phenomena, even though friendship selection and mating are processes of searching for compatible associates that occur over time. At the theoretical level, the concepts and categories normally used by social scientists to describe these phenomena have blurred edges and different gradations of membership (e.g., acquainted, friend), something that must be handled in a rigorous and systematic way, particularly when the aim is to build computational models of friendship and mating choice.

Researchers interested in modelling social dynamics have nowadays different methods and techniques to handle the complexity of this research field. This paper focuses on two of them. On one hand, agent-based modelling (from here on, ABM) is an outstanding method that enables us to study complex social dynamics. The simulation of an agent-based model facilitates the observation and analysis of such dynamics and the emergent selforganised processes that they imply. For instance, ABM has been applied for the study of opinion dynamics [7], cultural dynamics [8], language dynamics [9], the evolution of norms and culture [10-12], crowd behaviour [13] information diffusion [14], and many others. On the other hand, Fuzzy Logic is an excellent technique to handle the imprecise modes of reasoning in environments of uncertainty and vagueness, which is an usual feature in the social realm [15]. In fact, some of the attempts made to model social dynamics have already used fuzzy logic as an auxiliary tool for ABM. FLAME [16] introduces it in order to build an adaptive model for emotional behaviour, with important learning capabilities. Cioffi-Revilla [17] and afterwards J. G. Epstein et al. [18] make a call for the use of fuzzy logic in social simulation. J. M. Epstein and Axtell show some of its potential through the extension of the popular Sugarscape model [19] with fuzzy logic. Despite these early and promising attempts to integrate ABM and fuzzy logic, the work in this area has not developed much further.

Although in principle it may be thought that the use of fuzzy set theory makes agent-based modelling more elaborated, in practice it saves the modeller from taking some arbitrary decisions on how to use crisp values for representing properties that are inherently fuzzy. This prevents biases in the model implementation. The paper aims to show this advantage by exposing an example in which the consequences of applying fuzzy sets and operations to define a fuzzy friendship relationship are compared with a simpler implementation, with crisp values. Thus, one of the main contributions of this work paper is the definition of a process for the use and integration of ABM and fuzzy set theory as a mean to model friendship and partnership dynamics. To do so, the paper explores and work on an existing model that makes intensive use of social dynamics, named Mentat [20], which is introduced in Section 2. Fuzzy logic is presented in Section 3 as a helpful technique to deal with the kind of approximate or uncertain knowledge associated with modelling of social systems and, in more general terms, with the uncertainty inherent to this kind of systems. The paper illustrates this with the application of fuzzy logic to the Mentat model. Section 4 presents details on the communication among the neighbours and 
the way agents establish social relationships among them by taking into account their degree of similarity. The whole friendship dynamics is described in-depth in Section 5. Then, four versions of Mentat with different degrees of fuzzification are presented in Section 6 and a comparison of their performances is also provided, in order to evaluate the benefits of using fuzzy agents. Finally, Section 7 provides some concluding remarks derived from this analysis.

\section{Overview of Mentat Social Dynamics}

Mentat is an agent-based model that analyses the impact of demographics in the evolution of values in a society. Specifically, it has been applied to simulate the Spanish society from the period of 1980 to 2000, taking as a base data the European Values Study (EVS) [23]. In the Mentat model, social dynamics, in particular how friendship relationships and couples are formed, has an impact in the evolution of the demography and the transmission of values from one generation to another.

The Mentat model was programmed in Java using the Repast 3 framework [21]. It simulates 3019 agents along 1000 steps (20 years) + X steps of warmingup, with an $X=\{100 \rightarrow 1000\}$. It uses a torus-grid of $98 \times 98$ cells, with a fixed density of 3.2 cells / agent. Additional details concerning its theoretical foundations or technical details can be found in $[20,22]$. Mentat considers agents that are situated in a two-dimensions square grid world, and where agents can communicate with others in their neighbourhood, as explained in Section 4.1. A similarity function, which is described in Section 4.2, determines with which agents in the neighbourhood one can establish a friendship relationship. This idea is based on the the "proximity principle," which states that the more similar two individuals are, the stronger their chances of becoming friends are (for a throughout sociological analysis, see Section 5.1). Thus, the chances of two neighbours of becoming friends are directly proportional to their similarity. Moreover, if friendship is considered a gradual relationship, the more similar two agents are, the better friends they will become over time. This friendship process is summarised in Figure 1. There are several methods for quantifying this emergence and evolution of friendship, and the one selected for Mentat is described in Section 5.2.

The same as neighbours, friends can be also rated and ordered in terms of similarity, which is useful for the matchmaking process. First, friends are filtered taking into account Boolean questions such as "is this agent single?", "is it an adult?", "does it have opposite gender?". Afterwards, the selected subset are the "candidates" for becoming a spouse. The selection among the candidates is followed in the order defined by similarity: the first one of the queue who passes the filter will be chosen (see Section 5.3). This matchmaking process is summarised in Figure 2. As far as couples appear, so do families. Each couple will have chances of having some children following the demographic model criteria, which are specified by a set of probabilistic equations. For an insight into those decisions, outside of the scope of this paper, check [24].

\section{Introducing Fuzzy Sets in the Mentat Model}

The first version of the Mentat model was developed using crisp (nonfuzzy) values for agent attributes and relationships. This raised several issues, such as the lack of high similarity among the partners found (needed for an appropriate children generation) or 


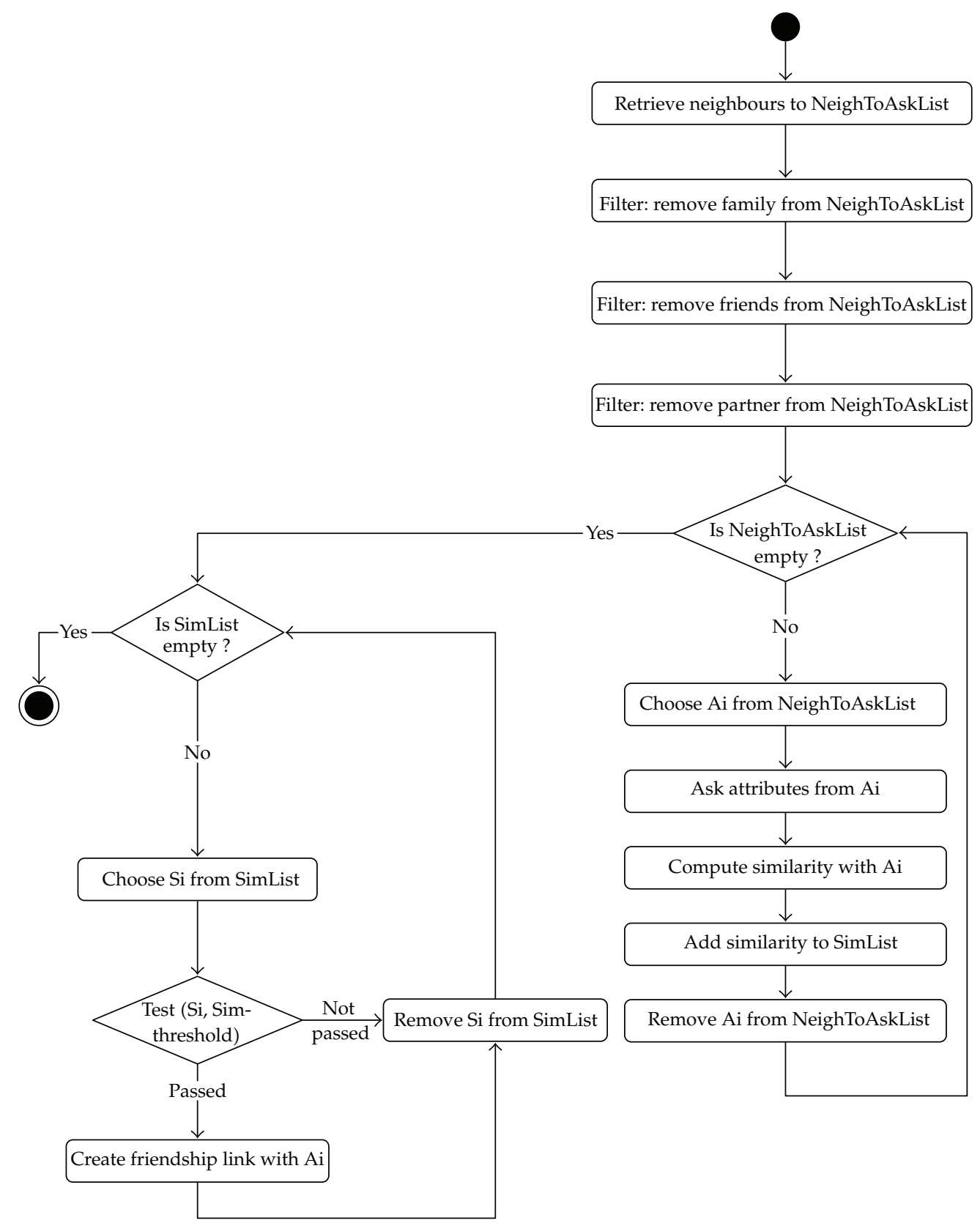

Figure 1: Flow diagram summarising the process of emergence of a friendship link.

the over-simplification of boolean and static friendship links, which is inconsistent with the literature. Fuzzy set theory has been introduced in the model in order to reduce these issues, avoid unnecessary biases, and produce more realistic results, consistent with the literature.

Here, we show the fuzzification process step by step, resulting in a fuzzy ABM, the final version of Mentat. The whole process consisted in the fuzzification of the agent 


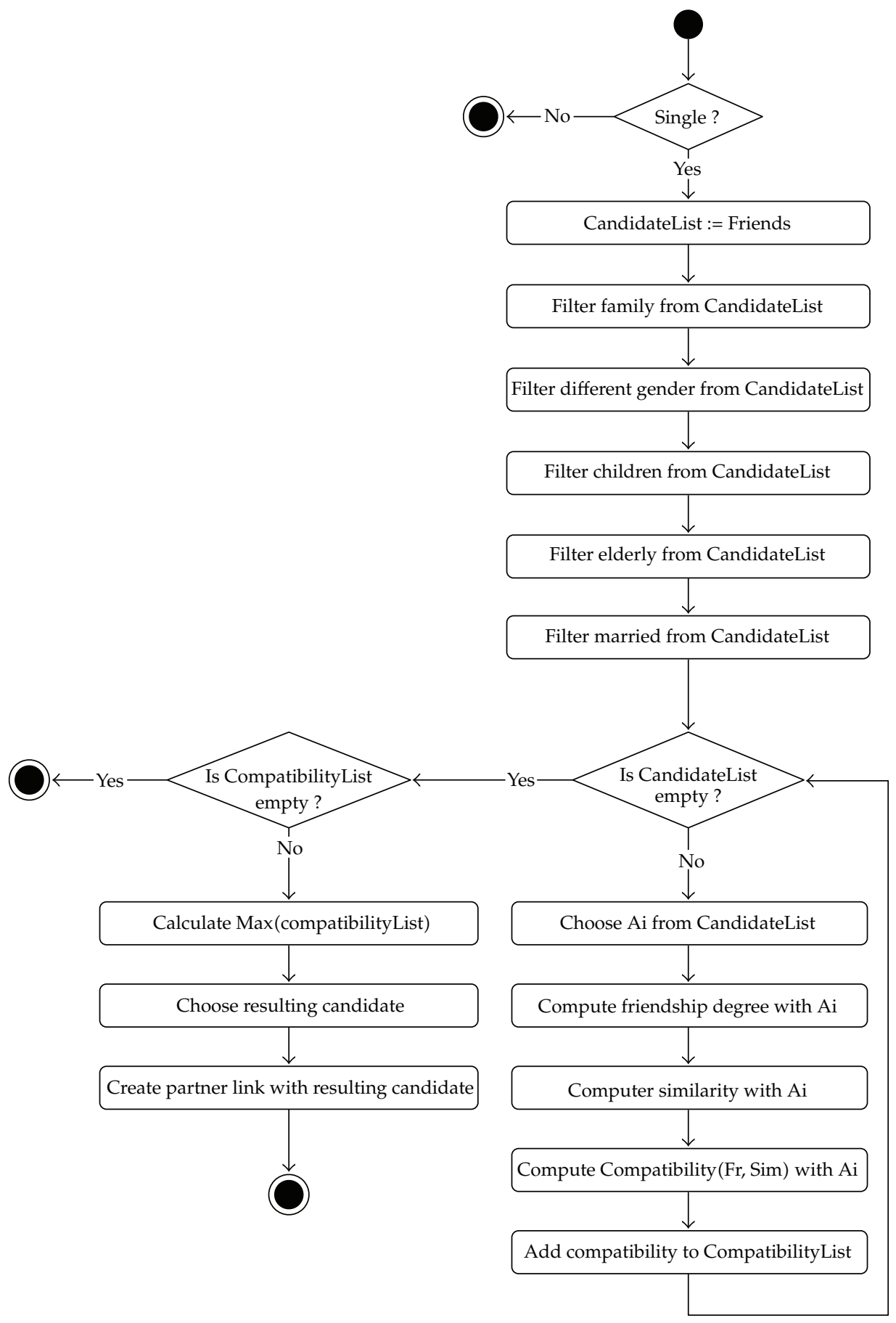

Figure 2: Flow diagram summarising the process of partner finding (matchmaking). 
characteristics, the similarity process, the fuzzification of the friendship relationship together with the introduction of a friendship function that varies over time, and a new partner matchmaking calculation. Comparing the results of a nonfuzzy and a fuzzy agent-based model, it can be assessed that the fuzzy version deals with the problem of similar partner finding in a more accurate way.

\subsection{Fuzzy Set Theory for Modelling}

Computational models of social systems can improve considerably by integrating fuzzy set theory. Firstly, because uncertainty is an inherent property of social systems, individuals are often vague about their beliefs, desires, and intentions and they evaluate their opportunities in vague and ever-changing contexts. Not surprisingly, they use linguistic categories with blurred edges and gradations of membership, such as "acquaintance" or "friend." Fuzzy logic is oriented at modelling imprecise modes of reasoning in an environment with uncertainty and vagueness $[25,26]$, as it happens for many aspects in human societies. Since vagueness is such a common feature in the social realm, fuzzy logic provides us with a useful way to handle this vagueness systematically and constructively [15]. Secondly, certain complex problems tackled by ABM cannot be properly modelled with the typical simple agent models. There are several ways of tackling such complexity, and depending on the context under study, one or another may be used. An example of a system that requires further considerations in agent modelling would be some sociological analysis derived from the European Values Study and the World Values Survey carried out by Inglehart [27]. In these surveys there are many questions about the degree of happiness, satisfaction in different aspects of life, or trust in several institutions. Although there is some kind of categorisation for the possible answers, such as "Very much" or "Partially," there is always some degree of imprecision, which is difficult to model with discrete/crisp categories. Even more, when the individual is evolving to different positions, some of these values get even more undefined. This issue arises also when modelling agent relationships such as friendship: is it possible to measure a degree of friendship between two persons? Similarity and friendship degree are blurry concepts, and this uncertainty must be modelled rigorously. In this context, an appropriate way of increasing the model complexity in order to improve its refinement can be fuzzy logic.

Finally, fuzzy logic might facilitate the communication and mutual understanding among different experts, something crucial in a multidisciplinary field like social simulation. For instance, it is common in ABM to take into account previous research carried out by social scientists and to consider their advice, which should be consulted repeatedly along the modelling process. Very often they use linguistic variables and terms to express their knowledge. These linguistic concepts could be better represented with fuzzy sets.

\subsection{Reviewing the Importance of Fuzzy Set Theory in ABM}

There is an increasing interest among social scientists for adding fuzzy logic to the social sciences toolbox [28]. Likewise, even though it is still incipient, there are several examples of researches linking fuzzy logic with social simulation. Since the early proposal of CioffiRevilla [17], in which the author gave preliminary arguments for the use of fuzzy logic in the social sciences and even computer simulations, some models followed this path. For instance, in some models, agents apply fuzzy logic rules to make their decisions; "fuzzy 
controls" or "fuzzy agents" are expert systems based on "If $\rightarrow$ Then" rules where the premises and conclusions are unclear. Unlike traditional multiagent models, where these completely determined agents are an oversimplification of real individuals, fuzzy agents take into account the stochastic component of the human behaviour.

Some authors have proposed to improve the agents' strategy choices within the iterated prisoner's dilemma using fuzzy logic decision rules [29]. Other researchers have claimed that simulation based on two-player games can use fuzzy strategies when analytic solutions do not exist or they are computationally complicated to obtain (because agents use fuzzy strategies; i.e., "If I think my opponent will choose action $x$, $I$ will choose action $\left.y^{\prime \prime}\right)$ [30]. Agent fuzzification has been already applied for social network analysis, as in the work of Carbó et al. [31], but considering mainly relationships among agents. Relevant examples of fuzzy multiagent based models are Fort and Perez work on spatial dilemmas [32], Jens Epstein's extension of the sugar-space model with fuzzy agents [18], and interesting computational modelling of "fuzzy love and romance" [33].

\subsection{Introducing Fuzzy Sets}

Given a universe of discourse $U$, a fuzzy set $\mu \in U \rightarrow[0,1]$ is a mapping function that gives a membership degree in the interval $[0,1]$ to every element of $U[25]$. Note that classical sets are particular cases of fuzzy sets. There are many human characteristics that do not have a clear boundary or depend on the interpretation or context, such as young or beauty. They can be represented by a fuzzy set on the set of human people, giving them a membership degree of the characteristic, such as, for instance, 0.8 or 0.2 . An example of such membership degree $\mu$ is represented in Figure 3, where $\mu$ (Age) can be observed. There, three fuzzy sets (i.e., functions) corresponding to three concepts are plotted, with a different membership degree depending on the age of the individual.

Given some fuzzy sets representing characteristics, sometimes it is needed to model algebraical operations on them, as "young" and "beauty," that must also be modelled, for example to trigger a rule in approximate reasoning systems. In order to use different operators, it is needed to define those algebras, generalising the classical sets. Therefore, a fuzzy relation $R$, generalising the classical logic relations, can be defined as $R: U \times U \rightarrow[0,1]$ [34]. Fuzzy relations have multiple applications to represent degrees of relations between objects in an universe. Those relations can also be characterised without a clear border, such as the Friendship relationship. Classical relations cannot express some types of information as "we are more or less friends," or "a little bit friends." Fuzzy relations are mostly used in artificial intelligence applications to represent degrees of similarity (that define unclear groups or clusters) or to model implications rules to make inference with uncertainty, imprecision, or lack of knowledge.

There are other definitions of fuzzy concepts related to the mathematical insight of the fuzzification process carried out here. See [35] for a formal clarification of basic operators ( $t$ norms and conorms), properties as $t$-transitivity or $t$-indistinguishability, and fuzzy similarity (generalising the classical equivalence relations [36]).

\section{Communication}

This section provides details on the local communication process among agents, based on a two-dimensional neighbourhood. Besides, it tackles the degree of similarity among each two 


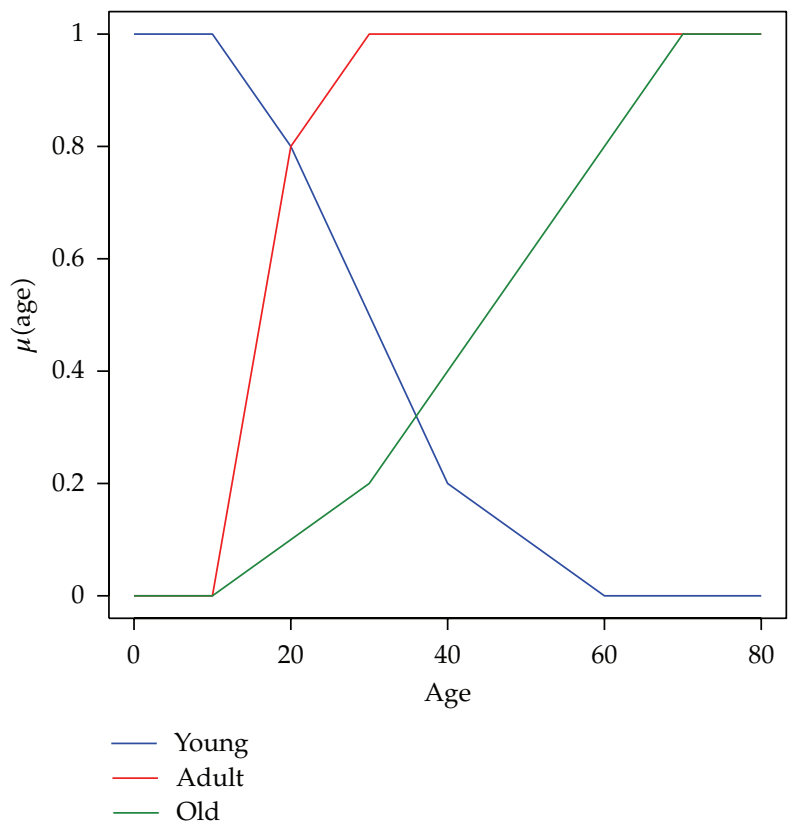

Figure 3: A graphical example of concept definitions in fuzzy terms.

neighbours, exposing two alternative ways of implementing it: a gratification algorithm and its improvement using fuzzy logic. This is illustrated with the Mentat model.

\subsection{Neighbourhood}

The world space in Mentat consists of a 2D square grid of size $100 \times 100$ configured as a torus, and each agent can be placed in a single grid cell. Agents cannot move, and grid cells are passive and all equal (but for their position in the grid). The space represents the "social space" of the agent, that is, the agents around agent $A$ are those with whom $A$ can relate, regardless of their actual geographical distance to it. That is, if $A$ lives in a city and it has no relation neither interaction with its building neighbours, they would not be in its social space. The same way, if $A$ has interaction with individuals who are geographically distant but that share an internet forum, they would be included, as there is chance of some sort of friendship to emerge among them.

As each agent has just local knowledge of such space, it is defined a neighbourhood for each, limiting the number of agents that anyone can interact with. Thus, an extended Moore neighbourhood of radius 6 was chosen, with a maximum of 168 agents around, and an average of 52 based on the agents density of 3.2, in order to reach the typical number of personal friends $[37,38]$. (Instead of considering the average of around 100 contacts that an individual usually has, the number of friends was chosen, as it reaches maximums of around 50. This is done for limiting agent interaction and avoiding a "town effect" in the model, in which the "friends of friends" of an agent would cover most of the population.)

As long as agent $A$ is a neighbour of agent $B$, they will be able to communicate. In some systems, agents need to compare themselves with their neighbours, in order to take decisions based on that new information (maybe selecting only some agents to establish 
further communication). This comparison is usually carried out through similarity measures. As explained in the next section, the basic crisp version of Mentat uses a discrete gratification similarity, while the fuzzy Mentat defines a new fuzzy similarity.

\subsection{Similarity Functions}

\subsubsection{Crisp Gratification Similarity}

The basic behaviour of a similarity function is that the greater the closeness among the two compared objects is, the greater the value it must return. In the case of Mentat, agents with a collection of characteristics are compared with each others. This function should determine how similar two individuals are, taking decisions grounded on sociological theory. Thus, it is obvious that some characteristics are sociologically more relevant than others to determine such similarity: for example, age is more important than hair colour (obviously not included in this model) or civil state (included). With the help of a domain expert, five main characteristics were chosen to determine the similarity: age, political ideology, religiosity, educational level, and economic status. This similarity will be used for the friendship emergence and for the spouse selection.

In the basic crisp Mentat, every function was defined as a crisp (nonfuzzy) function. Thus, similarity was not different and it was modelled and implemented through a not-normalised gratification algorithm. For each characteristic (from the chosen ones), it was defined a range of "closeness" (with three categories "close," "standard," and "far" distances). Depending on such "closeness" of the two characteristic values compared (e.g., the ideology of $A$ and the ideology of $B$ ), the similarity counter was gratified (in the "close" case), left equal ("standard") or penalised ("far"). The sum of all the results of the five attributes comparisons was the output of the function. Thus, this amount of points, could be compared with other results from other agents, and therefore determine which agent was more similar in a crude but straightforward way.

\subsubsection{Building a Fuzzy Similarity}

Mentat's gratification technique is not very sophisticated and could be improved. The use of fuzzy logic would significantly increase its accuracy. However, the use of fuzzy operators or functions needs the fuzzification of the attributes where they are applied. Therefore, fuzzy sets over these variables are formally defined (in a generalised manner).

Thus, the agent attributes, rather different from each other, were normalised in the real interval $[0,1]$ (each one depending on its original range). For example, let $\mu_{\text {wealthy }}$ : $U \rightarrow[0,1]$ be the fuzzy set that gives an economic grade based on the economy variable of the individual. This fuzzy set can be defined by segments with different growth (high class, middle class, working class, etc.) or by a linear function. This way, an individual with a $\mu_{\text {wealthy }}$ (ind) $=0.7$ would represent a person quite wealthy, with a good economic situation. Each fuzzy set would be defined by a similar process.

Afterwards, the fuzzy similarity can be defined using a $T$-indistinguishability, which generalises the classical equivalence relations. It can be obtained from the negation of a $T^{*}$-distance, where $T^{*}$ is the dual $t$-conorm of the $t$-norm $T$. A complete mathematical explanation beneath this can be found in Valverde [39], but roughly the distance between the attributes of the two agents compared is "how far they are," so its negation will point out 
"how similar they are." This way the aggregation of the similarities of each couple of fuzzy sets (by default, normalised) will return the total similarity rate among two individuals. The negation used is a fuzzy strong negation $N$ [40], the distance operator $d$ was defined as the difference of the fuzzy values, and the aggregation chosen is an ordered weighted averaging operator (OWA) [41]. Thus, the fuzzy relation is defined as follows:

$$
R_{\text {similarity }}(\text { ind }, \text { ind } 2)=\operatorname{OWA}\left(\forall \mu_{i} \in \text { ind }, N\left(d\left(\mu_{i}(\text { ind }), \mu_{i}(\text { ind } 2)\right)\right)\right)
$$

An OWA is a family of multicriteria combination (aggregation) procedures. By specifying suitable order weights (whose sum will result always 1) it is possible to change the form of aggregation: for example, the arithmetic average in the example OWA would need a value of 0.5 to both weights. The weights of the OWAs chosen in Mentat configuration will have, by default, standard average weights.

There are two reasons to justify why OWAs were used for the formalisation instead of standard average functions. First, the generalisation aspirations of this fuzzification process, so it could be applied in other ABMs, encourage the maximum level of abstraction. The possibility of choosing the most appropriate weights for each component could be useful to adapt to different problems (e.g., allowing different weights for each main attribute in the similarity definition). Second, this weight selection is allowed inside Mentat configuration: even if the main testing has been used using a single $T$ (Lukasiewicz) and standard weights, both can be tweaked to obtain different results or to focus on different phenomena.

\section{Friendship Dynamics}

This section addresses the sociological literature and empirical research about friendship dynamics. In particular, the important role played by social similarities in friendship choice is highlighted, especially when considering the "proximity principle," as explained. Then, this basic mechanism is formalised by proposing a logistic function, which allows to model over time the process by which strangers become acquaintances, and acquaintances are converted to friends and potentially to partners.

\subsection{Understanding Friendship}

Selecting a friend is among the most personal of human choices. However, friendship choice is also dependent on characteristics that are socially constructed. Consequently, it is not surprising that friendship groups tend toward social homogeneity. Members of the working class usually associate with other workers, and middle-class individuals generally choose friends who are middle class as well. Since individuals only have significant contact with others like themselves, most of the friends they make become similar in sociological, economical, and demographic terms. McPherson et al. remind us that classical Western thought already noticed this pattern in friendship dynamics: Aristotle pointed out in his Rhetoric and Nicomachean Ethics that people "love those who are like themselves" and Plato observed in his Phaedrus that "similarity begets friendship" [42, page 416].

Empirical research has confirmed this early conjectures: adult friendships are highly homogeneous in social and demographic statuses, race and ethnicity, beliefs, and interests, 
and that observed homogeneity is higher than it would be expected by considering randomchoice models [43]. In the context of social networks analysis, the homophily principle [42] has been identified as the mechanism bringing about this pervasive pattern of friendship choice $[1,44,45]$. These studies have established that similarity in social characteristics breed friendship connections among individuals (and accordingly, homophily implies that distance in terms of social characteristics translates into network distance). Individual preferences, however, must not be overemphasised. Individuals preferences can only affect the choices of associates within limited sets of available alternatives. Specifically, individuals tend to choose their friends from among those with whom they have regular contact in one or another of their focused activities (e.g., individuals choose a workplace for the job rather than for the coworkers' characteristics, but they might make friends in that context later on). Therefore, the structure of opportunities must be understood before one can estimate the importance of preference for similarity as a cause of observed homophily [46].

Once we consider these results, it becomes clear that social context structures friendship choice. Contextual theories of individual behaviour argue that (i) individual preferences and actions are influenced through social interaction and (ii) social interaction is structured by the social composition of the relevant environment [47]. The dynamic of "meeting" and "mating" by which strangers are converted to acquaintances, acquaintances to friends, and even maybe friends into partner follow the same rules. Meeting depends on opportunities alone (i.e., to be in the same place at the same time); instead, mating depends on both opportunities and attraction. How readily an acquaintance is converted to close friend depends on how attractive two people find each other and how easily they can get together.

The "proximity principle" indicates that the more similar two individuals are, the more likely they will meet and become friends [43]. Therefore, features like social status, attitudes, beliefs, and demographic characteristics - that is, degree of "mutual similarity" — channel individual preferences and they tend to show more bias toward homogeneous friendship choices.

\subsection{Strangers $\rightarrow$ Acquaintances $\rightarrow$ Friends: A Fuzzy Logistic Function}

Similarity, proximity, or friendship is vague or blurry categories, because they do not have clear edges. For this reason, a model of friendship dyads was developed, using the general framework presented above, but considering similarity and friendship as continuous variables. Thus, the fuzzy similarity will be used and friendship will be redefined as a fuzzy relationship. Besides, because friendship occurs through time, in Mentat it was modelled in dynamic terms, letting the friendship to evolve over time. Let $R_{\text {friend }}: U \times U \rightarrow[0,1]$ be the fuzzy relation on the set of individuals who have a degree of "friendship." This fuzzy relation gives a degree of friendship in the interval $[0,1]$ for every couple on individuals. Let Ind be an individual in $U$. The crisp set Friends (Ind) is defined as the set of all the individuals $x$ in $U$ whose $R_{\text {friend }}(\operatorname{Ind}, x)$ is greater than 0 . Therefore, every individual will have a range from true close friends to just "known" people with the rest of individuals. Note that some restrictions to this definition could be introduced in order to suit context needs.

The friendship process is conceived as a search for compatible associates, in terms of the proximity principle, and where strangers are transformed to acquaintances and acquaintances to friends as a continuous process over time. It is proposed the hypothesis 
that a logistic function [48] can describe formally the "friendship relationship" or degree of friendship for every couple of individuals. By using a logistic function, we want to express the intuitive dynamic of friendship, in which two similar individuals become friends faster than those individuals that do not share similar values or sociodemographical features (and friendship will hardly evolve between two totally dissimilar individuals). Therefore, a logistic model captures the core dynamic; that is, it reflects the likelihood that two individuals will become friends as a function of their individuals' traits. Although we highlight that this formalisation comes from an intuition (with no empirical research supporting it), we are confident that the general dynamics of friendship makes plausible the use of a logistic function, which has been extensively used in social research for measuring individuals' attitudes (such as logistic IRT models; see $[49,50]$ ). The following equation is proposed:

$$
\frac{d F}{d t}=g \times F_{(t)}\left(1-\frac{F_{(t)}}{K}\right)
$$

Equation (5.1) expresses the hypothesis that friendship increases over time. (In Mentat, friendship ties cannot be weakening nor broken. If two individuals are not similar enough, their tie will never grow strong, but once appeared it will always exist. This simplification was acceptable according to the domain expert advice.) Thus, at each point of time, $F_{(t)}$ defines the minimum degree of friendship that is given as an initial condition $(0<F(t)<K)$; $K$ is the maximum degree of friendship that agents can reach $(K$ can be understood as the level of "close friends"), and finally $g$ value defines the growth rate of friendship. However, this equation does not include the "proximity principle" described above. This principle can be included in (5.1) by modifying the growth rate $g$ and stating it as follows: the more similar in social characteristics two individuals are, the higher the growth rate of their friendship is. Therefore, $g$ needs to be made sensitive to the similarity value. Thus, the following equation can be expressed:

$$
g=R_{\text {similarity }}(\text { ind }, \text { ind } 2) \times J,
$$

where $R_{\text {similarity }}$ is a fuzzy-similarity measure between individual 1 and individual 2 defined in Section 4.2 (see (4.1)), and $J$ defines a multiplicative factor that increases the magnitude of $R_{\text {similarity }}$ within $g$. The objective of $J$ is turning $g$ more sensitive to $R_{\text {similarity }}$ values, and specially sensitive to high $R_{\text {similarity }}$ values. For this reason, $J$ describes an exponential growth depending on $R_{\text {similarity }}$ values. Therefore, $J$ itself is a function of $R_{\text {similarity }}$ that can be formalised as follows:

$$
J\left(R_{\text {similarity }}\right)=J_{0} \times e^{p \times R_{\text {similarity }}},
$$

where $J_{0}$ is the initial value of $J, p$ defines the constant of proportionality, and $R_{\text {similarity }}$ is the similarity value between the individuals. Some graphical examples and implementations of these formalizations are depicted in Figures 4 and 5. Thus, Figure 4 shows how the friendship will develop over time given different initial conditions, while Figure 5 depicts the exponential growth of $J$ as a function of the model fuzzy-parameter $R_{\text {similarity. }}$ (In Figure 4 , it is assumed that $K$ is equal to 1 and $F_{0}$ is equal to $0.01 ; g$ value is equal to $R_{\text {similarity }} \times J(s)$. On 


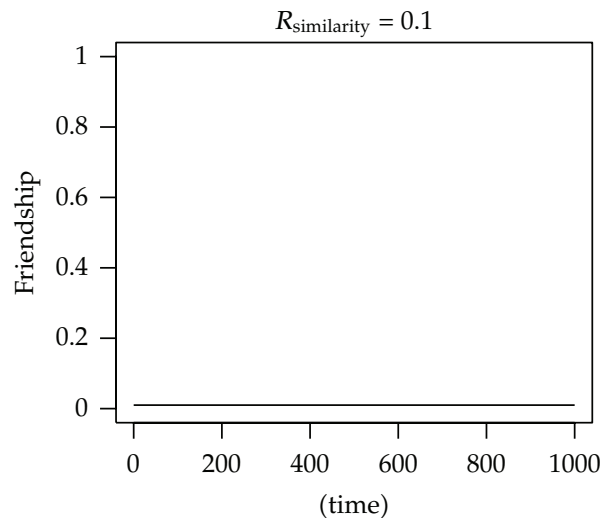

(a)

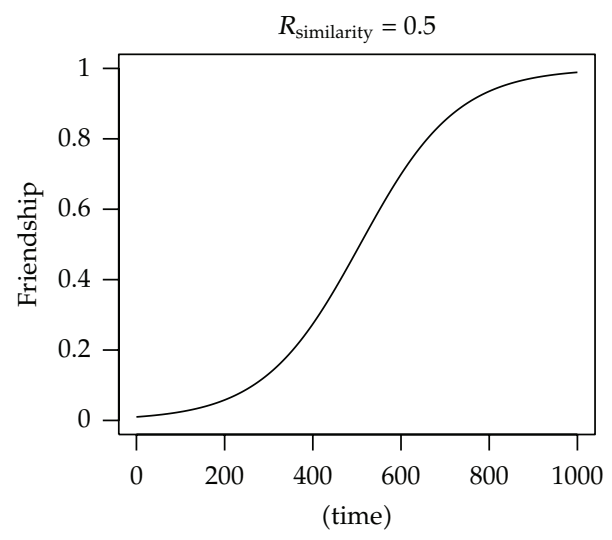

(c)

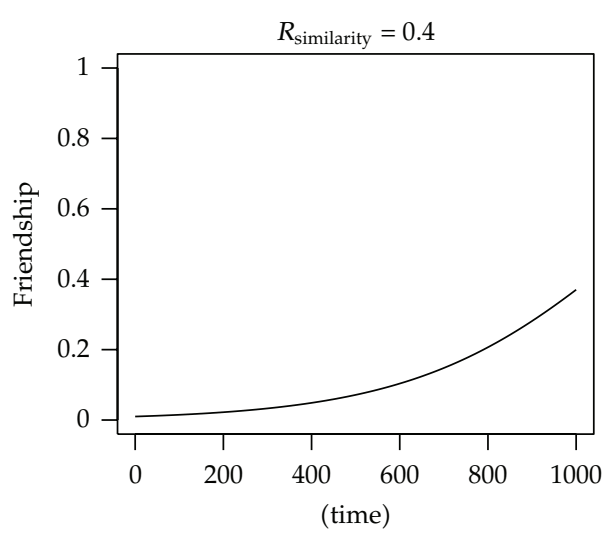

(b)

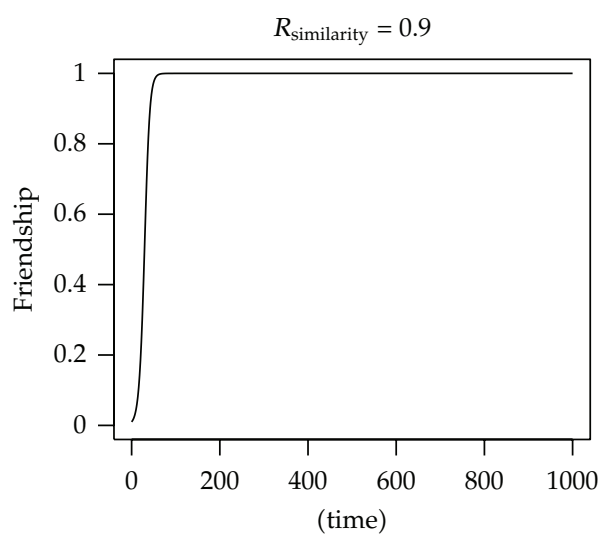

(d)

Figure 4: The logistic function chosen for the evolution of friendship, for several one-to-one $R_{\text {similarity }}$ values.

the other hand, in Figure 5, it is assumed that $p$ is equal to 5.8 and $J_{0}$ is equal to 0.001 . These constants were obtained by experimental procedures.)

\subsection{Friends $\rightarrow$ Partner}

The social system also simulates matchmaking. Once a couple is formed (e.g., via a marriage), a stable couple can be defined as a crisp relation: two persons are a couple or they are not. It is proposed to learn this classical relation $R_{\text {couple }}: U \times U \rightarrow\{0,1\}$ by using approximate reasoning and fuzzy inference techniques. Note that if a $R_{\text {couple }}$ relation is known, a "single" and "married" crisp set on $U$ is also known, defined as

$$
\begin{gathered}
\operatorname{married}(\text { ind })= \begin{cases}1, & \exists \text { ind }_{2} / R_{\text {couple }}\left(\text { ind }, \text { ind }_{2}\right)=1, \\
0, & \text { otherwise },\end{cases} \\
\text { single(ind) }=\text { NOT married(ind). }
\end{gathered}
$$




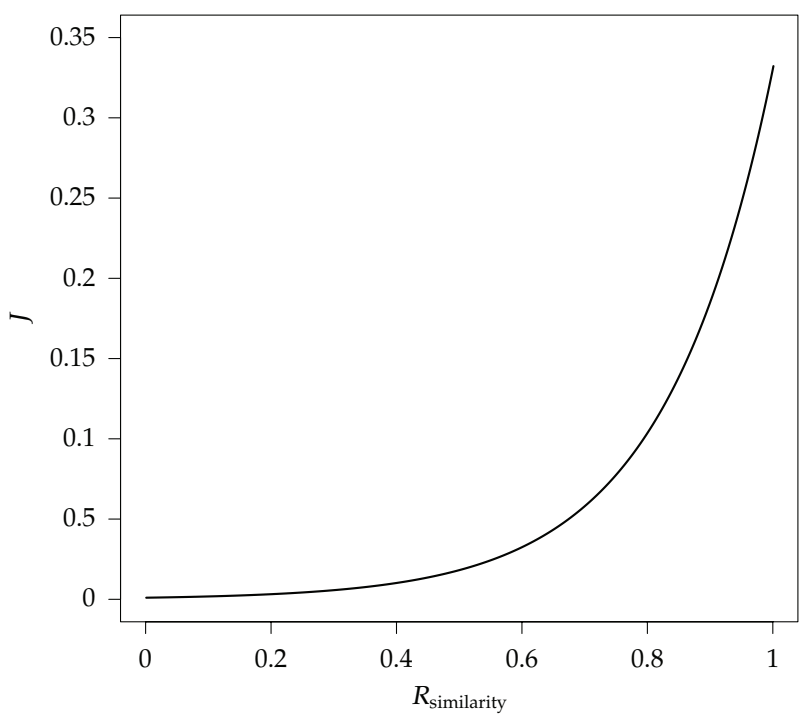

Figure 5: Exponential growth of parameter $J$, in relation with $R_{\text {similarity }}$.

It does not exist a specific process for finding a partner in previous social simulation models. A general definition for it was built up with the collaboration of a sociological domain expert. An agent will find a partner (always with a random possibility of failure not expressed in the mathematical definitions) between the friends that have different gender, are adults, and do not have a partner yet. The chosen one will be the "most compatible" one of its friends, where compatibility is defined as the aggregation of how strong their friendship is and how similar they are (this is an oversimplification with respect to real life, and it is future work to allow some randomness in this process).

This important information of how couples are established can be obtained by inferring a fuzzy relation $R_{\text {compatible }}: U \times U \rightarrow[0,1]$, which in turn can be expressed using a fuzzy aggregation operator [41], and operations on the classical set "has no partner": $U \rightarrow\{0,1\}$, the fuzzy set "adult": $U \rightarrow[0,1]$, the classical relation "has different gender": $U \times U \rightarrow\{0,1\}$, and one fuzzy rule of inference, where the premise is the conjunction of the classical sets.

An OWA is again applied to formally define the $R_{\text {compatible }}: U \times U \rightarrow[0,1]$ fuzzy relation using the $R_{\text {similarity }}: U \times U \rightarrow[0,1]$ and the $R_{\text {friend }}: U \times U \rightarrow[0,1]$ fuzzy relation, as the following mapping:

$$
\begin{array}{r}
R_{\text {compatible }}(\text { Ind }, \text { Ind } 2):=\operatorname{OWA}\left(R_{\text {friend }}(\text { Ind }, \text { Ind } 2), R_{\text {similarity }}(\text { Ind }, \text { Ind } 2)\right) \\
=w_{1} * R_{\text {friend }}(\text { Ind, Ind } 2)+w_{2} * R_{\text {similarity }}(\text { Ind, Ind } 2) \\
\forall \text { Ind, Ind } 2 \text { in } U, \text { where } w_{1}+w_{2}=1 .
\end{array}
$$




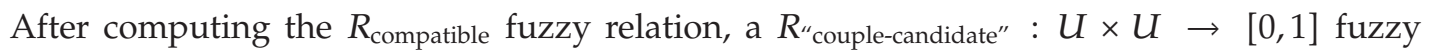
relation can be computed by using the "single" set and the "Adult" fuzzy set on $U$ as follows:

$$
R_{\text {couple-candidate }}\left(\text { Ind,Ind2) }:= \begin{cases}R_{\text {compatible }}(\text { Ind, Ind2) } & \text { if Adult(Ind) AND } \\ & \text { Adult(Ind2) AND } \\ & \text { single(Ind) AND } \\ & \text { single(Ind2) } \\ 0 & \text { otherwise. }\end{cases}\right.
$$

Note that the AND conjunction is implemented by a $t$-norm. It can be done many times because of the associative property of $t$-norms. Thus, $T(x, y, z)=T(T(x, y), z)$. Then, the method to marry an individual Ind is to find Ind2 such that it maximises the fuzzy relation $R_{\text {"couple-candidate" }}$ (Ind, Ind2). Then, it would be possible to compute $R_{\text {couple }}$ (Ind, Ind2) $:=1$.

\subsection{Family}

As a result of the friendship dynamics exposed in the previous subsections, agents can find spouses to match with. Those couples may have children, following certain restrictions and demographic equations (for an in-depth view of those, check [24]). Thus, it produces the emergence of families with parents, children, and brotherhood. The family is represented as a different group type. No third-level relationships are considered (e.g., grandparents, uncles, and cousins) in order to focus on the family nuclei. Those nuclei are localised and aggregated in space, as children tend to appear close to their parents. This process promotes the appearance of clusters in the social network, where the new-born agents will relate with the friends of their family, relating to the friends of the brothers or parents and even to their children. This link has not been fuzzified, as it was not found necessary.

\section{Results and Discussion}

In order to assess the relevance of fuzzy sets in the model, this section analyses the results of the execution of the different versions of Mentat. As shown in Table 1, four different implementations, each one with two configurations, have been analysed, focusing on three measures. The fuzzy modifications have been grouped in two main ones: "Fuzz-Sim," when the attributes are normalised and the similarity operator fuzzified, as stands Section 4.2; and "Fuzz-Fri," when the friendship turns to be fuzzy, evolving over time, and affecting the partner choice (Section 5.2). The four ABM represent all the possible combinations between these, represented in the Table 2 in the pair (Fuzz-Sim, Fuzz-Fri). Thus, there is a

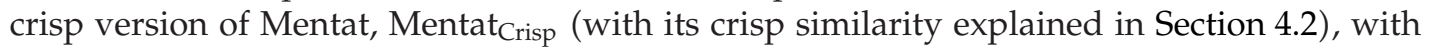
no fuzzy properties; the Mentat FuzzSim, simply the same model but with the "Fuzz-Sim"; the Mentat ${ }_{\text {FuzzFri }}$ with "Fuzz-Fri" but not "Fuzz-Sim"; last Mentat ${ }_{\text {FuzzAll, with all the fuzzy }}$ modifications (i.e., both Fuzz-Sim and Fuzz-Fri).

The two configurations deal with two possible ways of friendship emerging: one promoting random friends (and therefore an agent can be linked to a nonsimilar neighbour) and the other promoting similarity-based friends (and therefore an agent will rarely be linked 
Table 1: Four different implementations of Mentat.

\begin{tabular}{lcc}
\hline & Crisp similarity & Fuzzy similarity \\
\hline Crisp friendship & Mentat & Mentat $_{\text {FuzzSim }}$ \\
Fuzzy friendship & Mentat $_{\text {FuzzFri }}$ & Mentat $_{\text {FuzzAll }}$ \\
\hline
\end{tabular}

Table 2: Comparison among the different ABM, in increasing order of fuzzification.

\begin{tabular}{|c|c|c|c|c|}
\hline & $\begin{array}{c}\text { Mentat }_{\text {Crisp }} \\
(0,0)\end{array}$ & $\begin{array}{c}\text { Mentat }_{\text {FuzzSim }} \\
(1,0)\end{array}$ & $\begin{array}{c}\text { Mentat }_{\text {FuzzFri }} \\
(0,1)\end{array}$ & $\begin{array}{c}\text { Mentat }_{\text {FuzzAll }} \\
(1,1)\end{array}$ \\
\hline \multicolumn{5}{|l|}{ Config. random friendship } \\
\hline Mean couple $R_{\text {Similarity }}$ & $0.76^{*}$ & 0.77 & $0.76^{*}$ & 0.77 \\
\hline Mean couple $R_{\text {Friendship }}$ & $(* *)$ & $(* *)$ & $0.72^{*}$ & 0.80 \\
\hline Mean couple $R_{\text {Compatibility }}$ & $0.76^{*}$ & 0.77 & $0.74^{*}$ & 0.79 \\
\hline \multicolumn{5}{|l|}{ Config. similar friendship } \\
\hline Mean couple $R_{\text {Similarity }}$ & $0.73^{*}$ & 0.77 & $0.73^{*}$ & 0.78 \\
\hline Mean couple $R_{\text {Friendship }}$ & $(* *)$ & $(* *)$ & $0.54^{*}$ & 0.76 \\
\hline Mean couple $R_{\text {Compatibility }}$ & $0.73^{*}$ & 0.77 & $0.64^{*}$ & 0.77 \\
\hline
\end{tabular}

to a nonsimilar neighbour, as it will give priority to the most similar ones). This is not a trivial decision, because the friendship evolution function already deals with similarity, and if a neighbour is not similar at all, it will never be more than an acquaintance. It is not evident if the closer way to real world is giving double strength to similarity (in the second option) or letting randomness to decide who will be the friend (and thus maybe ignoring similar people). It has to be mentioned that none of the two configurations is so deterministic and both are based on probabilities.

The parameters analyse the couples and how they are affected by the changes in the configuration and fuzzification. The $R_{\text {Similarity }}$ shows the proximity taking into account all the characteristics of each partner in a couple. The $R_{\text {Friendship }}$ focuses on the friendship link between them, which in a way (according to the logistic function) depends on their similarity too, but also in the time spent together. The $R_{\text {Compatibility }}$ is taken as an average of the other two. The values have been obtained after averaging the output of several executions of each version. Thus, in every execution, it is the mean of the property in every couple. These averaged values are shown in Table 2 in order to compare the performance of the different implementations.

As the first two models have a Boolean friendship, their compatibility is always the same as the similarity. In the first configuration, when the friendship is rarely involved in the neighbours linking, the similarity rates are noticeably similar in all the versions. However, in the second one it is clear that the ones with fuzzy similarity slightly increase their success. But the bigger changes can be observed in the friendship: Mentat ${ }_{\text {FuzzAll }}$ beats the other versions with a greater $R_{\text {Similarity }}$ and $R_{\text {Friendship }}$ in both configurations. We compared the four different orders of fuzzification described above for both $R_{\text {Similarity }}$ and $R_{\text {Friendship }}$ of couples, by using the statistical test "one-way analysis of variance" for unrelated samples. By using this statistical test, we want to detect evidence of significant differences among the four model implementations. The first analysis revealed significantly higher degrees of similarity between the couples according to the degree of fuzzification of the model, with the 
Fisher's statistical significance test equal to $7.281(3 ; 8,814)$, and a $P$-value $P<.001$. Similarly, the second analysis revealed significantly higher degrees of friendship between the couples, $F(3 ; 8,814)=5,247.735, P<.001$. These results provide strong evidence against the null hypothesis, namely, that the difference in the means among the four orders of fuzzification are by chance, both for $R_{\text {Similarity }}$ and $R_{\text {Friendship }}$. These results approach the theoretical qualitative assessments made by the sociological friendship theory considered $[43,47]$ and the domain expert. Intuitively, the couples of Mentat ${ }_{\text {FuzzAll }}$ are more similar to each other (following the proximity principle) and take into account the degree of friendship in the process of choosing partner (which implies historical evolution of the relationship).

\section{Concluding Remarks}

This paper has exposed Mentat's social dynamics in-depth, detailing the social relationship mechanisms and how the social network emerges. Besides, throughout the different sections, a fuzzification process was carried out, redefining several crisp model concepts as fuzzy concepts and relations. Fuzzy sets theory improved the behaviour of the ABM in those aspects with some degree of uncertain knowledge.

Thus, some concepts of social relationship dynamics were explained, including an evolution function that was applied for the changing of friendship over time. Fuzzy logic was introduced step by step. Therefore, fuzzy sets over each agent attribute were defined, together with a new fuzzy similarity operator that would influence friendship emergence and partner choice. The friendship relationship nature and importance in the model was significantly modified, fuzzifying it, making it evolve using a logistic function, and letting it influence in the partner choice as much as the similarity rate. The results of these changes were discussed and considered positive, as far as they improve the proximity to the qualitative assessments of the theory.

To sum up the fuzzy application, a sociological friendship theory has been exposed. It was searched where it can be applied, found the useful tools to do that, implemented the application, and extracted a collection of results that are used to validate the model against the theory.

Future research lines that could be followed could take into account other interesting friendship theories. There are deep studies in homophily in social networks [42] that could be implemented. An aspect that the Mentat model ignores but it is important enough to be considered is the stability of friendship [51]. Besides, the fuzzy Mentat could be extended to analyse the importance of weak links along one's life, a new possibility that the crisp Mentat did not allow [52].

\section{Acknowledgments}

The authors acknowledge support from the project Agent-based Modelling and Simulation of Complex Social Systems (SiCoSSys), supported by the Spanish Council for Science and Innovation, with Grant TIN2008-06464-C03-01. They also thank the support from the Programa de Creación y Consolidación de Grupos de Investigación UCM-BSCH, GR35/10-A.

\section{References}

[1] P. F. Lazarsfeld and R. K. Merton, "Friendship as a social process: a substantive and methodological analysis," in Freedom and Control in Modern Society, M. Berger and T. Abel, Eds., Van Nostrand, New York, NY, USA, 1954. 
[2] P. S. Bearman, J. Moody, and K. Stovel, "Chains of affection: the structure of adolescent romantic and sexual networks," American Journal of Sociology, vol. 110, no. 1, pp. 44-91, 2004.

[3] P. S. Bearman and H. Brückner, "Promising the future: virginity pledges and first intercourse," American Journal of Sociology, vol. 106, no. 4, pp. 859-912, 2001.

[4] J. O. G. Billy, K. L. Brewster, and W. R. Grady, "Contextual effects on the sexual behavior of adolescent women," Journal of Marriage and Family, vol. 56, no. 2, pp. 387-404, 1994.

[5] K. L. Brewster, "Neighborhood context and the transition to sexual activity among young black women," Demography, vol. 31, no. 4, pp. 603-614, 1994.

[6] Y. Åberg, "The contagiousness of divorce," in The Oxford Handbook of Analytical Sociology, P. Hedström and P. S. Bearman, Eds., pp. 342-364, Oxford University Press, Hampshire, UK, 2009.

[7] G. Deffuant, F. Amblard, G. Weisbuch, and T. Faure, "How can extremism prevail? a study based on the relative agreement interaction model," Journal of Artificial Societies and Social Simulation, vol. 5, no. 4, 2002.

[8] H. Petter and G. Andreas, "Modelling the dynamics of youth subcultures," Journal of Artificial Societies and Social Simulation, vol. 8, no. 3, p. 3, 2005.

[9] E. Marchione, M. Salgado, and N. Gilbert, "“"What did you say?" Emergent communication in a multiagent spatial configuration," Advances in Complex Systems, vol. 13, no. 4, pp. 469-482, 2010.

[10] R. Axelrod, "An evolutionary approach to norms," The American Political Science Review, vol. 80, no. 4, pp. 1095-1111, 1986.

[11] R. Axelrod, "The dissemination of culture: a model with local convergence and global polarization," Journal of Conflict Resolution, vol. 41, no. 2, pp. 203-226, 1997.

[12] C. A. Caldwell and A. E. Millen, "Studying cumulative cultural evolution in the laboratory," Philosophical Transactions of the Royal Society B, vol. 363, no. 1509, pp. 3529-3539, 2008.

[13] J. M. Epstein, "Modeling civil violence: an agent-based computational approach," Proceedings of the National Academy of Sciences of the United States of America, vol. 99, no. 3, pp. 7243-7250, 2002.

[14] M. H. Chang and J. E. Harrington, "Discovery and diffusion of knowledge in an endogenous social network," American Journal of Sociology, vol. 110, no. 4, pp. 937-976, 2005.

[15] M. J. Smithson and J. Verkuilen, Fuzzy Set Theory: Applications in the Social Sciences, Sage Publications, 1st edition, 2006.

[16] M. Seif El-Nasr, J. Yen, and T. R. Ioerger, "FLAME—fuzzy logic adaptive model of emotions," Autonomous Agents and Multi-Agent Systems, vol. 3, no. 3, pp. 219-257, 2000.

[17] C. A. Cioffi-Revilla, "Fuzzy sets and models of international relations," American Journal of Political Science, vol. 25, no. 1, pp. 129-159, 1981.

[18] J. G. Epstein, M. Mohring, and K. G. Troitzsch, "Fuzzy-logical rules in a multi-agent system," in SimSocVI Workshop, p. 25, Groningen, The Netherlands, 2003.

[19] J. M. Epstein and R. Axtell, Growing Artificial Societies: Social Science from the Bottom Up, The Brookings Institution, Washington, DC, USA; The MIT Press, Cambridge, Mass, USA, 1996.

[20] S. Hassan, L. Antunes, and J. Pavón, "Mentat: a data-driven agent-based simulation of social values evolution," in Multi-Agent-Based Simulation X, vol. 5683 of Lecture Notes in Artificial Intelligence, pp. 135-146, 2010.

[21] M. J. North, N. T. Collier, and J. R. Vos, "Experiences creating three implementations of the Repast agent modeling toolkit," ACM Transactions on Modeling and Computer Simulation, vol. 16, pp. 1-25, 2006.

[22] J. Pavón, M. Arroyo, S. Hassan, and C. Sansores, "Agent-based modelling and simulation for the analysis of social patterns," Pattern Recognition Letters, vol. 29, no. 8, pp. 1039-1048, 2008.

[23] R. Inglehart, University Consortium for Political, I., Research, S.: World Values Surveys and European Values Surveys 1981-1984, 1990-1993, and 1995-1997. Inter-University Consortium for Political and Social Research, 2000.

[24] S. Hassan, L. Antunes, and M. Arroyo, "Deepening the demographic mechanisms in a data-driven social simulation of moral values evolution," in Multi-Agent-Based Simulation IX, vol. 5269 of Lecture Notes in Artificial Intelligence, pp. 46-59, 2009.

[25] L. A. Zadeh, "Fuzzy sets," Information and Computation, vol. 8, pp. 338-353, 1965.

[26] L. A. Zadeh, Fuzzy Sets, Fuzzy Logic, and Fuzzy Systems. Selected Papers by Lotfi A. Zadeh, vol. 6 of Advances in Fuzzy Systems-Applications and Theory, World Scientific, River Edge, NJ, USA, 1996. 
[27] A. P. Norris and R. Inglehart, Sacred and Secular, Cambridge University Press, Cambridge, UK, 2004.

[28] A. Katz, M. vom Hau, and J. Mahoney, "Explaining the great reversal in Spanish America: fuzzy-set analysis versus regression analysis," Sociological Methods \& Research, vol. 33, no. 4, pp. 539-573, 2005.

[29] B. Lomborg, "Nucleus and shield: the evolution of social structure in the iterated prisoner's dilemma," American Sociological Review, vol. 61, no. 2, pp. 278-307, 1996.

[30] J. E. West and B. Linster, "The evolution of fuzzy rules as strategies in two-player games," Southern Economic Journal, vol. 69, no. 3, pp. 705-717, 2003.

[31] J. Carbo, J. M. Molina, and J. Dávila, "Fuzzy referral based cooperation in social networks of agents," AI Communications, vol. 18, no. 1, pp. 1-13, 2005.

[32] H. Fort and N. Perez, "The fate of spatial dilemmas with different fuzzy measures of success," Journal of Artificial Societies and Social Simulation, vol. 8, no. 3, 2005.

[33] H. Situngkir, "The ribbon of love: fuzzy-ruled agents in artificial society," Tech. Rep. BFI Working Paper Series, 2007.

[34] L. A. Zadeh, "Fuzzy sets as a basis for a theory of possibility," Fuzzy Sets and Systems, vol. 1, no. 1, pp. 3-28, 1978.

[35] S. Hassan, L. Garmendia, and J. Pavón, "Introducing uncertainty into social simulation: using fuzzy logic for agent-based modelling," Reasoning-Based Intelligent Systems, vol. 2, no. 2, pp. 118-124, 2010.

[36] L. A. Zadeh, "Similarity relations and fuzzy orderings," Information Science, vol. 3, pp. 177-200, 1971.

[37] L. Hamill and N. Gilbert, "Social circles: a simple structure for agent-based social network models," Journal of Artificial Societies and Social Simulation, vol. 12, no. 2, p. 3, 2009.

[38] L. Hamill, Communications, travel and social networks since 1840: a study using agent-based models, Ph.D. thesis, University of Surrey, Surrey, UK, 2010.

[39] L. Valverde, "On the structure of F-Indistinguishability operators," Tech. Rep., Computer Science Division, University of California at Berkeley, Berkeley, Calif, USA, September 1984.

[40] B. Schweizer and A. Sklar, Probabilistic Metric Spaces, Dover Publications, New York, NY, USA, 2005.

[41] R. R. Yager, "Families of OWA operators," Fuzzy Sets and Systems, vol. 59, no. 2, pp. 125-148, 1993.

[42] M. McPherson, L. Smith-Lovin, and J. M. Cook, "Birds of a feather: homophily in social networks," Annual Review of Sociology, vol. 27, pp. 415-444, 2001.

[43] L. M. Verbrugge, "The structure of adult friendship choices," Social Forces, vol. 56, no. 2, pp. 576-597, 1977.

[44] D. B. Kandel, "Homophily, selection, and socialization in adolescent friendships," The American Journal of Sociology, vol. 84, no. 2, pp. 427-436, 1978.

[45] K. Yamaguchi, "Homophily and social distance in the choice of multiple friends: an analysis based on conditionally symmetric Log-Bilinear association model," Journal of the American Statistical Association, vol. 85, no. 410, pp. 356-366, 1990.

[46] S. L. Feld, "Social structural determinants of similarity among associates," American Sociological Review, vol. 47, no. 6, pp. 797-801, 1982.

[47] R. R. Huckfeldt, "Social contexts, social networks, and urban neighborhoods: environmental constraints on friendship choice ( Detroit)," American Journal of Sociology, vol. 89, no. 3, pp. 651-669, 1983.

[48] P. Blanchard, R. L. Devaney, and G. R. Hall, Differential Equations, Brooks Cole, 2nd edition, 2002.

[49] R. J. Mislevy, "Bayes modal estimation in item response models," Psychometrika, vol. 51, no. 2, pp. 177-195, 1986.

[50] S. K. Balasubramanian and W. A. Kamakura, "Measuring consumer attitudes toward the marketplace with tailored interviews," Journal of Marketing Research, vol. 26, no. 3, pp. 311-326, 1989.

[51] B. Wellman, R. Y. L. Wong, D. Tindall, and N. Nazer, "A decade of network change: turnover, persistence and stability in personal communities," Social Networks, vol. 19, no. 1, pp. 27-50, 1997.

[52] P. Csermely, Weak Links: Stabilizers of Complex Systems from Proteins to Social Networks, Springer, 1 edition, 2006. 


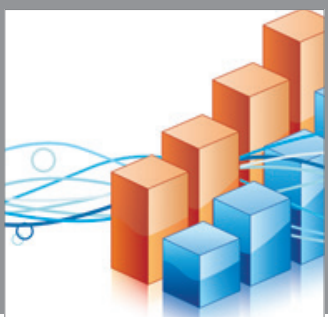

Advances in

Operations Research

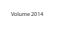

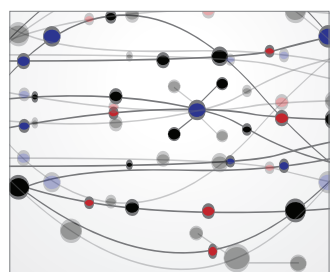

\section{The Scientific} World Journal
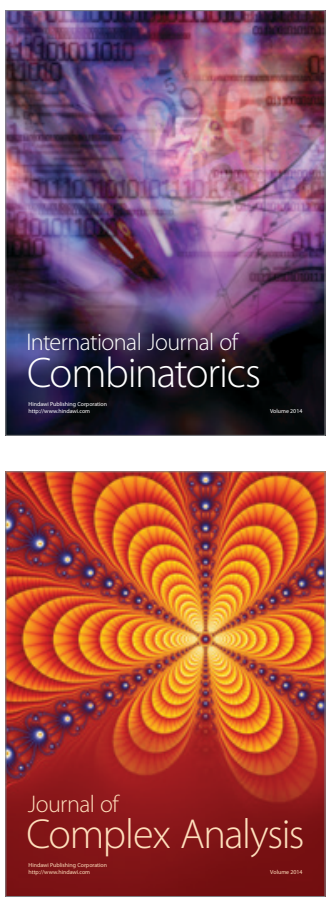

International Journal of

Mathematics and

Mathematical

Sciences
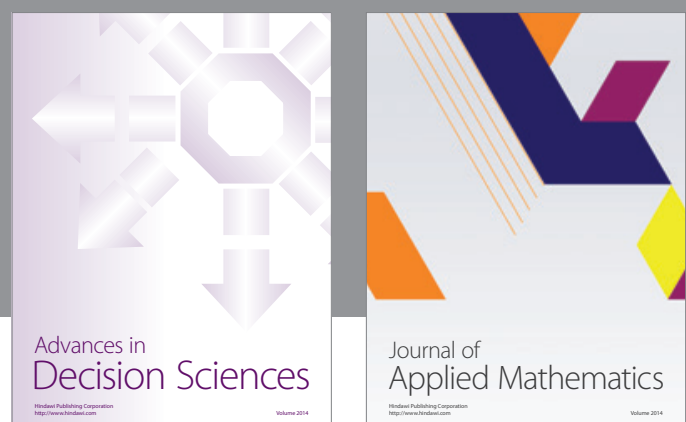

Journal of

Applied Mathematics
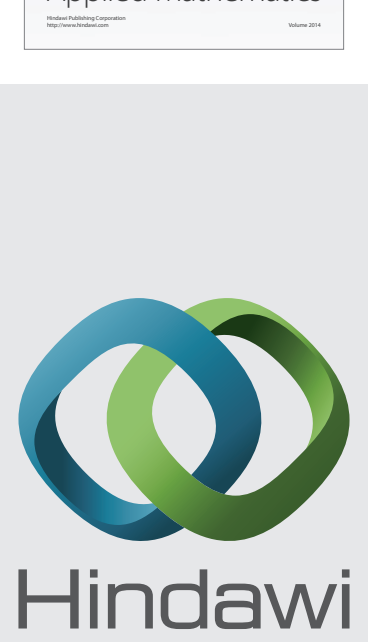

Submit your manuscripts at http://www.hindawi.com
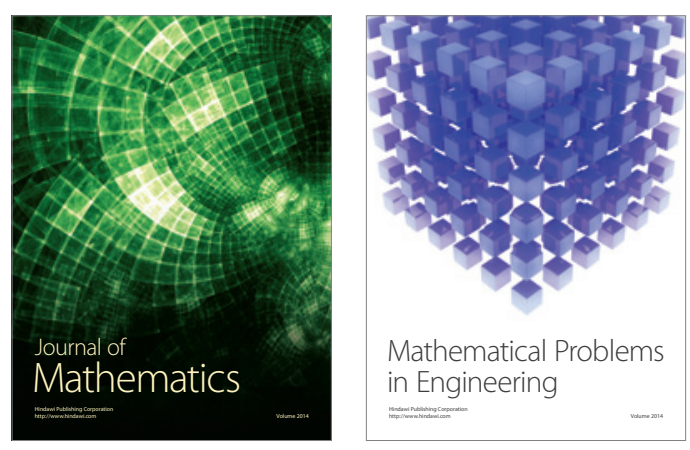

Mathematical Problems in Engineering
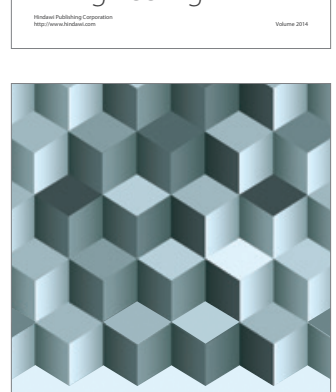

Journal of

Function Spaces
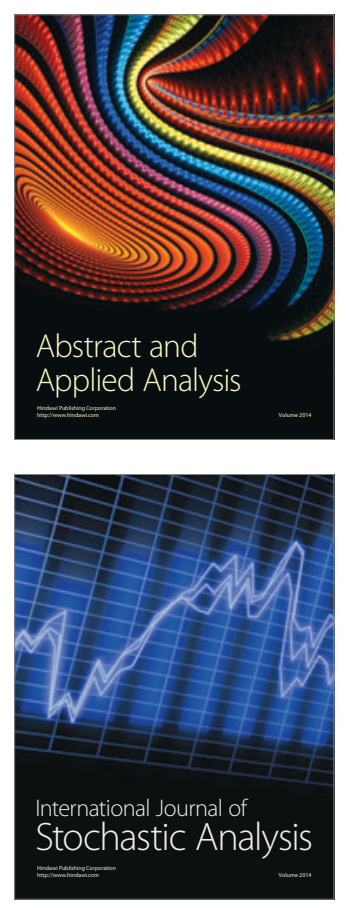

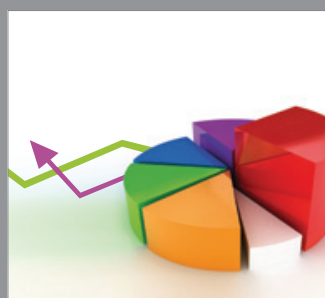

ournal of

Probability and Statistics

Promensencen
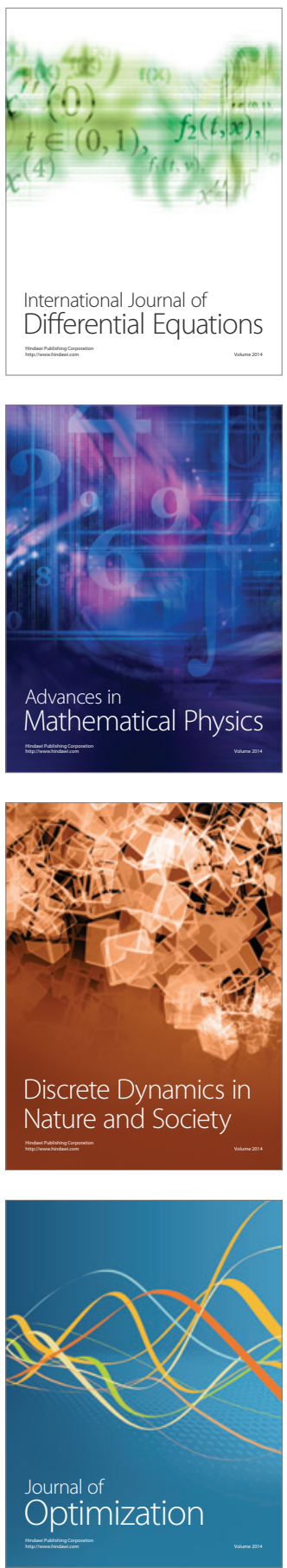